\title{
Isolation and identification of toxigenic fungi from foodstuffs at Sohag Governorate, Egypt
}

\author{
Zohri A.A. ${ }^{1}$, Saber S.M. ${ }^{2}$, Youssef M.S. ${ }^{2}$, Marwa Abdel-Kareem M. ${ }^{2}$ \\ ${ }^{1}$ Botany \& Microbiology Department, Faculty of Science, Assiut University, Egypt. \\ ${ }^{2}$ Botany Department, Faculty of Science, Sohag University, Egypt.
}

Rec. 1 Mar, 2014 Accept. 2 Apr, 2014

\begin{abstract}
The common fungi contaminated different 25 foodstuffs collected from Sohag Governorate were isolated and their ability to produce mycotoxins were examined. A total of 150 fungal isolates belonging to 57 species in addition to two species varieties of 13 genera were isolated and identified. The genus Aspergillus was represented by 23 species in addition to two species-varieties followed by Penicillium which was represented by 11 species. The genus Fusarium was represented by 6 species followed by Alternaria (4), Mucor (3), Trichoderma (2) and Achremonium ( 2 species). The remaining 6 genera were represented by only one species for each genus. All the 150 collected fungal isolates were screened for their ability to produce mycotoxins. The results indicated that 53 out of the 150 fungal isolates (35\%) were able to produce one or more of eight mycotoxins (aflatoxin, ochratoxin, fuminisin, sterigmatocystin, patulin, zearalenone, alternariol and diacetoxyscirpenol).
\end{abstract}

Key words: food sources, moulds, mycotoxins.

\section{Introduction}

Mycobiota are found in a wide range of environments due to their capability to utilize a variety of substrates and to their relative tolerance to low $\mathrm{pH}$, low water activity, and low temperature (Huis in't Veld, 1996). Generally, foods have essential nutrients for fungal growth, thus fungi can appear and spoil different foods. Fungal spoilage of food causes economic losses worldwide (Dantigny et al., 2005). In addition, some fungi synthesize mycotoxins, which can be a hazard for human health. Pitt (1996) defined mycotoxins as fungal metabolites that when ingested, inhaled or absorbed through the skin cause illness on human and animal death.

Mycotoxin is produced during the growth of fungi and can be found out or in the hyphae and spores of these organisms (Zucchi and Melo, 2009; Köppen et al., 2010). If ingested, mycotoxins may cause acute or chronic disease episodes, termed mycotoxicosis (Köppen et al., 2010; Medeiros et al., 2012). Mycotoxin long-term exposure has also been related to several mycotoxicosis, such as carcinogenic, mutagenic, teratogenic, estrogenic, hemorrhagic, immunotoxic, nephrotoxic, hepatotoxic, dermotoxic neurotoxic and immunosuppressive (Richard, 2007; Medeiros et al., 2012).

Currently, more than 500 different mycotoxins have been discovered and this number do not stop increasing. Among the most economically and toxicologically important mycotoxins that pose greatest potential risk to human and animal health as food and feed contaminants are: aflatoxins, strigmatocystin, trichothecenes, fumonisins, zearalenone, ochratoxin, alternariol, patulin, and certain ergot alkaloids (CAST, 2003; Bennett and Klich, 2003; Richard, 2007; Köppen et al., 2010).

The aim of this study was designed to isolate, collect and identified common fungi contaminated different foodstuffs in Sohag Governorate, Egypt. Also, the ability of the collected fungal isolates for mycotoxins production was aimed.

\section{Materials and Methods \\ Collection of Different Food Samples:}

A suitable weight or volume of samples as sources for food borne fungi of different 25 foodstuffs including fruits (bananas, lemon, orange, pomegranate, strawberry, tangerine and tomato), canned juices (apple,

* Corresponding author:
Dr. Zohri A.A.


guava, orange and pineapple), fresh juices (orange and tomato), seeds or grains (broad bean, lentils, maize, millet, peanut, phaseolus, rice and wheat) and others (carrot, luncheon, potato and spud) were collected from different shops and markets at Sohag Governorate, Egypt. The samples were brought to the laboratory and kept in a refrigerator $\left(3-5^{\circ} \mathrm{C}\right)$ till isolation of fungi.

\section{Isolation and idintification of fungi:}

Isolation of fungi was made by using the dilution plate method (Tournas et al., 2006) or direct plate method (Pitt et al., 1992) according to the nature of samples. The medium used for isolation of fungi was modified Czapeks Dox agar medium with a pH 6.5 (Al-Doory, 1980). The plates were incubated at $28 \pm^{\circ} \mathrm{C}$ for 7-10 days. Purified fungal isolates were identified based on macro- and microscopic characteristics on standard media according to different identification references (Reper and Fennell, 1965; Ainsworth, 1971; Ellis, 1976; Booth, 1977; Pitt, 1979; Klich and Pitt, 1992; Moubasher, 1993; Domsch et al., 2007; Pitt and Hocking, 2009).

Screening of selected fungal isolates for mycotoxin formation:

A total of 150 different fungal isolates collected from different foodstuffs during this study belonging to Aspergillus, Penicillium, Fusarium, Alternaria, Mucor, Trichoderma, Acremonium, Stachybotrys, Ulocladium, Drechslera, Trichurus and Neurospora were examined for their ability to produce mycotoxins. All fungal isolates were cultivated on potato-dextrose broth (200g potato\& $20 \mathrm{~g}$ dextrose per liter of distilled water) at $28 \pm^{\circ} \mathrm{C}$ for 10 days as static cultures. Only Fusarium and Stachybotrys cultures were then incubated at $10{ }^{\circ} \mathrm{C}$ for another 10 days. At the end of incubation, each culture was homogenized for five minutes in a high speed blender $(1600 \mathrm{rpm})$ with double volume of chloroform. The chloroform extracts were washed with equal volume of distilled water, dried over anhydrous sodium sulphate, filtered then concentrated to near dryness and purified by column chromatography (AOAC, 1980). Mycotoxins were identified by thin layer chromatographic technique on precoated silica gel plate $60 \mathrm{~F}_{254}$ (Merck) as described by (El-Kady and Moubasher, 1982).
Different mycotoxins were identified by comparison with reference standards (Scott et al., 1970; Schroeder and Kelton, 1975; Gimno, 1979).

\section{Results and Discussion}

Fungi are able to grow on different kinds of food: cereals, meat, milk, fruit, vegetables, nuts, fats and their products. The fungal growth may result in several kinds of food-spoilage, off-flavours, toxins, discolouration, rotting and formation of pathogenic or allergenic propagules (Chelkowski, 1991; Tipples, 1995; Filtenborg et al., 1996). Toxigenic fungi, under favorable conditions could produce mycotoxin in foods. Mycotoxins are very hazardous to human health. As dangerous metabolites, their clinical effect range between carcinogenic (aflatoxins), estrogenic (zearalenone), nephratoxic (ochratoxin A), neurotoxic (fuminisin B) dermatotoxic (trichothecenes) and immunosuppressive (aflatoxin $\mathrm{B}_{1}$ ). Mycotoxins have four basic kinds of toxicity: acute, chronic, mutagenic and teratogenic. The occurrence of fungi in different foodstuffs collected from the shops and markets of different sanitation levels in Sohag Governorate were identified and tested for their ability to produce mycotoxins. A total of 57 species in addition to two species varieties belonging to 13 genera of filamentous fungi were isolated and identified. Genus Aspergillus came first by 23 species in addition to two speciesvarieties followed by Penicillium (11 species) as shown in table (1). These results are in agreement with those obtained by (Njobeh et al., 2009). who revealed the predominance of Aspergillus and Penicillium with $96 \%$ of food samples.

The genus Fusarium was represented by 6 species followed by Alternaria (4 species), Mucor (3), Trichoderma (2) and Achremonium (2). The remaining 6 genera were represented by only one species for each genus (Table, 1). Similarly, (Toffa et al. 2013). revealed the occurrence of a large number of fungi in food commodities from the Republic of Niger. They found that among the most important genera: Aspergillus (in 63\% of samples), Fusarium (9.7\%) and Penicillium (3.3\%).

The most common species of Aspergillus were A. flavus and A. flavus var. columnaris. 
They isolated with high frequencies (16 and 12 out of 25 tested foodstuffs, respectively (Table, 1). (Njobeh et al., 2009). reported that the predominance species from the genus Aspergillus was Aspergillus flavus (in $85 \%$ of maize and peanuts). (Ruadrew et al., 2013) studied the mycobiota of different food commodities (Jasmine brown rice, long grain rice, fragrant rice, peanut, black bean, black pepper, crushed chilli and five spice powder) and revealed the occurrence of various genera of fungi from which: Aspergillus spp. (55\%), Penicillium spp. (15\%), Rhizopus spp. (11\%), Mucor spp. (3\%), Monascus spp. (1\%) and Eurotium spp. (1\%). All of fungal species isolated in this study were also isolated from different food sources in Egypt (Youssef, 1986; Zohri et al., 1992; 1995; El-Maghraby et al., 1995; Abdel-Kareem, 2010; Obied-Allah; 2011).

The 150 collected fungal isolates were screened for their ability to produce mycotoxins. The results indicated that 53 isolates $(35 \%)$ were able to produce eight mycotoxins (Aflatoxins, Ochratoxins, Fuminisins, Sterigmatocystin, Patulin, Zearalenone, Alternariol and Diacetoxyscirpenol) as shown in table (2). Twenty-seven out of 38 isolates of A. flavus group were recorded as aflatoxin producers (71\%). Three out of 19 A. flavus isolates produced the four aflatoxins: $B_{1}, B_{2}, G_{1} \&$ $\mathrm{G}_{2}$. Aflatoxin $\mathrm{B}_{1}$ was produced by 8 out of 14 A. flavus var. columnaris isolates, 1 out of 19 A. flavus, 1 out of 2 A. flavo-forcatis and 2 out of 3 A. parasiticus. . Aflatoxins $\mathrm{B}_{1} \&$ $\mathrm{B}_{2}$ were produced by 3 isolates (one of each of A. flavus, A. flavus var. columnaris and $A$. parasiticus). Aflatoxins $\mathrm{B}_{1} \& \quad \mathrm{G}_{1}$ were produced by 6 out of 19 A. flavus isolates and 2 of 14 A. flavus var. columnaris. Aflatoxins $\mathrm{G}_{1} \& \mathrm{G}_{2}$ were produced by only one isolate of A. flavus (Table, 2).

(Alborch et al., 2012). recorded that $31 \%$ (13 out of 42) of A. flavus strains and the two $A$. parasiticus strains isolated from maize were able to produce aflatoxins. It has been estimated that only about $30-40 \%$ of known isolates of A. flavus produce aflatoxins and virtually all isolates of $A$. parasiticus are toxigenic (Varga et al., 2011; Diner and Davis 1966). reported that 23 isolates of $A$. flavus out of 26 produced aflatoxin $\mathrm{B}_{1}$. (ElMaraghy and Zohri, 1988). recorded the production of aflatoxin by two out of four tested isolates of A. flavus var. columnaris. (Sanchez-Hervas et al., 2008). reported that $64.1 \%$ of 120 tested A. flavus had the ability to produce aflatoxin B. Aflatoxins have been detected in various food commodities from many parts of the world (Ariño et al., 2009; Reddy et al., 2009). and are presently considered as one of the most dangerous contaminants of food and feed. Aflatoxin $\mathrm{B}_{1}$ is the most potent. Aflatoxins are immunosuppressive, carcinogenic, teratogenic, and mutogenic (Miller and Wilson, 1994).

In the present study, ochratoxins A \& B were produced by the three tested isolates of A. ochraceus group (Table, 2). Ochratoxin is primarily a kidney toxin but in sufficiently high concentration it can damage the liver as well. It is a carcinogen in rats and mice and is suspect as the causative agent of human disease (Richard, 2007). Patulin was produced by 8 out of 17 Penicillium isolates examined in this study. Patulin has been reported to be mutagenic, naurotoxic, genotoxic, immuntoxic and able to cause gastrointestinal effects in rodents but little is know about its carcinogenic effect in the case of human beings (Delage et al., 2003).

Two tested isolates of Aspergillus nidulans and $A$. caespitosus have the ability to produce sterigmatocystin (Table, 2). Production of sterigmatocystin by Aspergillus nidulans is agrees with earlier results. (Schroeder and Kelton, 1975) and (El-Maraghy and Zohri, 1988). found that all the isolates tested of Aspergillus nidulans (= E. nidulans) produced this toxin. Also, E. nidulans was recorded as sterigamatocystin producer in several other studies (Bennett et al., 1989; Zohri and Ismail, 1994). Sterigmatocystin was reported to be both hepatotoxic and carcinogenic (Lillehoj and Ciegler, 1968).

Zearalenone, in the present study, was produced by 2 isolates of Stachybotrys chartarum, in addition to one of each of Acremonium strictum and Fusarium semitectum. Fuminisin was produced by one isolate of Fusarium solani. Only one isolate of Fusarium reticulatum was able to produce diacetoxyscirpenol. The genus Fusarium contains important mycotoxin-producing species that have been implicated in human diseases (Marasas et al., 1984; Nelson et al., 1993). Alternariol was produced by 6 
isolates of Alternaria spp. and one Drechslera rostrata. It has been suggested that A. alternata might be one of the etiological factors for human esophageal cancer in Linxian, China (Dong et al., 1987). Alternariol and A1ternariol monomethyl ether are mutagenic (Schrader et al., 2001).

In conclusion, the present results forward an evidence of potential hazard to human health in Sohag Governorate may exist due to the presence of large number of toxigenic fungi in different foodstuffs consumed in this area. Also, owing to the adverse effect of mycotoxins, it is necessary to continue monitoring the toxigenic fungi in different foodstuffs to safeguard the health of consumers and to generate data for future planning of food safety issues. Also, precautions must be adopted during processing, handling, transporting and storage different foodstuffs to avoid contamination by toxigenic fungi.

\begin{tabular}{|c|c|c|c|c|c|c|c|c|c|c|c|c|c|}
\hline Source & Bananas & $\begin{array}{c}\text { Broad } \\
\text { bean }\end{array}$ & $\begin{array}{l}\text { Canned } \\
\text { apple } \\
\text { juice }\end{array}$ & $\begin{array}{l}\text { Canned } \\
\text { guava } \\
\text { juice }\end{array}$ & $\begin{array}{l}\text { Canned } \\
\text { orange } \\
\text { juice }\end{array}$ & $\begin{array}{c}\text { Canned } \\
\text { Pineapple } \\
\text { juice }\end{array}$ & Carrot & $\begin{array}{c}\text { Fresh } \\
\text { orange } \\
\text { juice }\end{array}$ & $\begin{array}{l}\text { Fresh } \\
\text { Tomato } \\
\text { juice }\end{array}$ & Lemon & Lentils & Lunchoan & Maize \\
\hline Acremonium & - & - & + & - & - & - & - & - & - & - & - & - & - \\
\hline Acremonium rutilum Gams & - & - & & - & - & - & - & - & - & - & - & - & - \\
\hline Acremonium strictum Gams & - & - & + & - & - & - & - & - & - & - & - & - & - \\
\hline Alternaria & - & - & - & - & - & - & - & + & + & - & - & - & - \\
\hline A. alternata (Fries) Keissler & - & - & - & - & - & - & - & + & + & - & - & - & - \\
\hline A. brassicicola (Schw.) Wiltshire & - & - & - & - & - & - & - & + & - & - & - & - & - \\
\hline A. longipes (Ellis and Everch.) Mason & - & - & - & - & - & - & - & - & - & - & - & - & - \\
\hline A. solani Ellis and Martin & . & . & . & . & . & . & . & . & + & . & . & . & . \\
\hline Aspergillus & + & + & + & + & + & + & + & + & + & + & + & + & + \\
\hline A. awamorii Nakazawa & - & - & - & + & - & - & - & - & - & - & + & - & + \\
\hline A. caespitosus Raper and Thom & - & - & - & - & - & - & - & - & - & - & - & - & - \\
\hline A. ficuum (Reich.) Hennings & - & - & - & + & - & + & - & - & - & - & - & + & - \\
\hline A. flavo-forcatis Batista and Maia & - & - & - & - & - & - & - & - & - & - & - & + & + \\
\hline A. flavus Link & + & + & - & + & + & - & - & - & + & + & + & + & + \\
\hline $\begin{array}{l}\text { A. flavus var. columnaris Raper \& } \\
\text { Fennel }\end{array}$ & + & - & - & ++ & ++ & - & - & + & + & + & + & + & + \\
\hline $\begin{array}{l}\begin{array}{l}\text { A. foetidus (Nakazawa) Thom and } \\
\text { Raper }\end{array} \\
\end{array}$ & - & - & - & - & - & - & + & - & - & + & - & - & - \\
\hline A. foetidus var. pallidus Nakazaw & - & - & - & + & - & - & - & - & - & - & - & - & - \\
\hline A. fumigatus Fresenius & - & - & - & - & - & - & - & - & - & + & - & - & - \\
\hline A. granulosus Raper and Thom & - & - & - & - & - & . & - & - & - & + & - & . & - \\
\hline A. heteromorphus Batista and Maia & + & - & - & - & - & - & - & - & - & - & - & - & - \\
\hline A.japonicus Saito & - & - & - & - & - & - & - & - & - & - & - & - & - \\
\hline A. nidulans (Eidam) Wint. & - & - & - & - & - & - & - & - & - & - & - & - & - \\
\hline A. niger Van Tieghem & - & - & - & - & - & - & - & - & + & - & - & + & - \\
\hline A. ochraceus Wilhelm & - & - & - & - & - & - & - & - & - & + & - & - & - \\
\hline A. parasiticus Speare & - & - & - & - & - & - & - & - & - & - & - & + & - \\
\hline A. phonicis (Cda.) Thom & - & - & + & - & - & - & - & - & . & . & - & - & - \\
\hline A. pulverulentus (McAlpine) Thom & - & - & - & - & - & - & - & - & - & + & - & - & - \\
\hline A. pulvinus Kwon and Fennell & - & - & - & - & - & - & - & - & 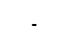 & - & - & - & - \\
\hline A. sclerotiorum Huber & - & - & - & - & - & - & - & - & - & - & - & - & - \\
\hline A. sulphureus (Fres.) Thom and Church & - & - & - & - & - & - & - & - & - & + & - & - & - \\
\hline $\begin{array}{l}\begin{array}{l}\text { A. sydowii (Bain \& Sart) Thom and } \\
\text { Church }\end{array} \\
\end{array}$ & + & - & - & - & + & - & - & - & + & - & - & + & - \\
\hline A. terreus Thom & - & - & + & - & + & - & - & - & + & - & - & - & - \\
\hline A. tubingensis (Schöber) Mosseray & + & - & + & - & + & - & - & - & - & + & - & + & - \\
\hline A. versicolor (Vuillemin) Tiraboschi & . & . & . & + & . & . & . & . & . & . & . & . & . \\
\hline Drechslera & - & - & - & - & - & - & - & - & - & + & - & - & - \\
\hline D. rostrata Richardson and Fraser & - & - & - & - & - & - & - & - & - & + & - & - & - \\
\hline Fusarium & - & - & - & - & - & - & + & + & - & - & - & - & + \\
\hline F. equiseti (Corda) Saccardo & - & - & - & - & - & - & + & - & - & - & - & - & - \\
\hline
\end{tabular}

Table (1): Different fungal isolates collected and identified in this study from different foodstuffs in Sohag Governorate, Egypt. 


\begin{tabular}{|c|c|c|c|c|c|c|c|c|c|c|c|c|c|}
\hline Source & Bananas & $\begin{array}{c}\text { Broad } \\
\text { bean }\end{array}$ & $\begin{array}{c}\text { Canned } \\
\text { apple } \\
\text { juice }\end{array}$ & $\begin{array}{c}\text { Canned } \\
\text { guava } \\
\text { juice }\end{array}$ & $\begin{array}{l}\text { Canned } \\
\text { orange } \\
\text { juice }\end{array}$ & 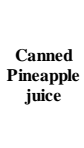 & Carrot & $\begin{array}{c}\text { Fresh } \\
\text { orange } \\
\text { juice }\end{array}$ & $\begin{array}{c}\text { Fresh } \\
\text { Tomato } \\
\text { juice }\end{array}$ & Lemon & Lentils & Lunchoan & Maize \\
\hline $\begin{array}{l}\text { F. proliferatum (Matsushima) } \\
\text { Nirenberg }\end{array}$ & & & & & & & & & & & & & - \\
\hline F. reticulatum Montagne & & & & & & & & & & & & & \\
\hline $\begin{array}{l}\begin{array}{l}\text { F. semitectum Berkeley and } \\
\text { Ravenel }\end{array} \\
\end{array}$ & - & - & - & & - & & & + & & & - & & - \\
\hline F. solani (Mart.) Saccardo & & & & & & & & + & & & & & + \\
\hline F. verticillioides Sacc. Nirenberg & & & & & & & & & & & & & + \\
\hline Mucor & + & & & & & & & & & & & + & \\
\hline M. circinelloides Van Tieghem & . & . & . & . & . & - & . & . & . & . & . & - & . \\
\hline M. hiemalis Wehmer & - & - & - & . & - & - & - & . & . & - & . & - & . \\
\hline M. racemosus F resenius & + & . & - & - & . & - & - & . & . & . & . & + & . \\
\hline Neurospora & - & + & - & - & . & - & - & - & - & - & - & - & - \\
\hline N. crassa Shear and Dodge & . & + & - & & . & & & . & & & . & & . \\
\hline Penicillium & + & - & - & & - & + & + & . & . & + & . & + & . \\
\hline P. chrysogenum Thom & - & & & - & & - & & & . & + & & & - \\
\hline P. corylophilum Dierckx & + & . & - & - & . & - & & . & . & & . & & . \\
\hline P. cyclopium Westling & + & - & . & - & - & - & - & $\therefore$ & - & - & - & - & $\therefore$ \\
\hline$P$. digitatum $(\mathbf{P e r s}$. Ex Fr.) Sacc. & . & . & - & - & . & . & - & . & . & . & . & . & . \\
\hline P. duclauxxi Delacroix & . & - & . & & . & + & . & . & & . & . & . & . \\
\hline P. expansum Link ex Gray & + & . & - & . & . & . & . & . & & + & . & . & . \\
\hline p. italicum Wehmer & - & - & - & - & - & - & - & . & . & + & . & . & . \\
\hline P. pupurogenum Stoll & . & - & - & . & . & . & & . & . & & . & & . \\
\hline P. rugulosum Thom & & - & - & & . & & + & . & & & . & & \\
\hline P. oxalicum Currie and Thom & & & & & & & & & & & & & \\
\hline P. vermiculatum Dangeard & & & & & & & & & & & & + & \\
\hline Rhizopus & & & & & & & & & & & & & \\
\hline R. oryzae Went \& Geerlings & . & . & . & & . & & . & . & & . & . & . & . \\
\hline Stachybotrys & & & & & & & & & & & & & \\
\hline S. chartarum (Ehrenb.) Hughes & & & & & & & & & & & & & \\
\hline Trichoderma & & & & & + & & & & & & & & \\
\hline T. hamatum (Bonord.) Bain & . & & & - & + & . & & & . & & & & . \\
\hline T. gansus yumenensis & . & . & - & & . & & . & . & & 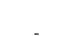 & . & . & . \\
\hline Trichurus & & & & & & & & & & & & & \\
\hline T. spiralis Hasselbr & & & & & & & & & & & & & \\
\hline Ulocladium & & & & & & & & & & & & & \\
\hline U. atrum Preuss & . & . & . & . & . & . & - & 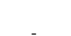 & & 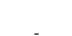 & 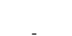 & . & . \\
\hline Total genera (13) & 3 & 2 & 2 & 1 & 2 & 2 & 3 & 3 & 2 & 3 & 1 & 3 & 2 \\
\hline Total species (57) & 8 & 2 & 4 & 4 & 5 & 2 & 3 & 4 & 6 & 12 & 2 & 9 & 5 \\
\hline Total varieties (2) & 1 & . & - & 2 & 1 & & & 1 & 1 & 1 & 1 & 1 & 1 \\
\hline
\end{tabular}

Table (1): continued. 
Journal of Environmental Studies [JES] 2014. 13: 1-12

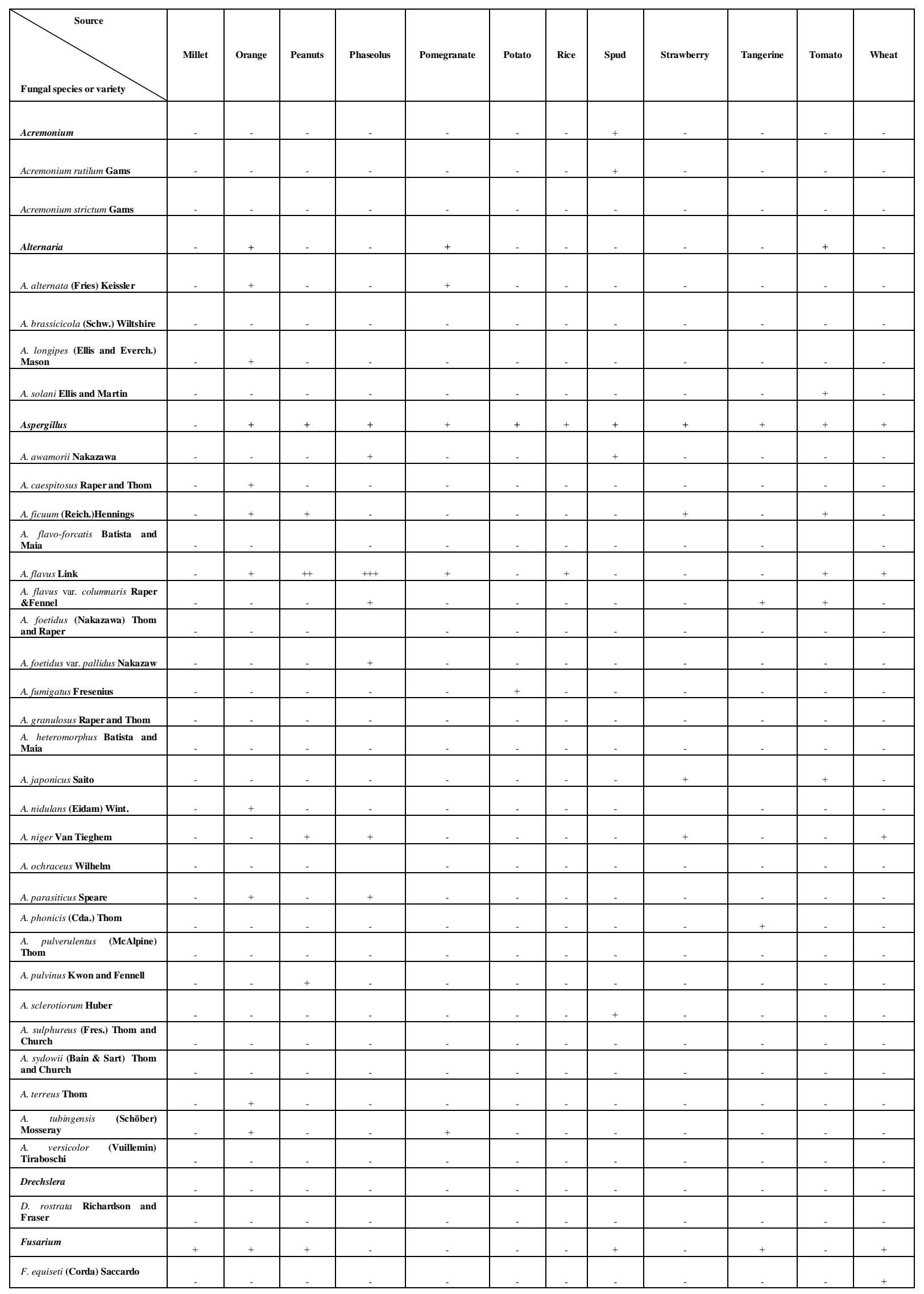

Table (1): continued. 
Journal of Environmental Studies [JES] 2014. 13: 1-12

\begin{tabular}{|c|c|c|c|c|c|c|c|c|c|c|c|c|}
\hline Source & Millet & Orange & Peanuts & Phaseolus & Pomegranate & Potato & Rice & Spud & Strawberry & Tangerine & Tomato & Wheat \\
\hline $\begin{array}{l}F \text {. proliferatum (Matsushima) } \\
\text { Nirenberg }\end{array}$ & - & - & - & - & - & - & - & + & - & - & - & - \\
\hline F. reticulatum Montagne & - & - & + & - & & & - & & & 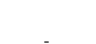 & - & - \\
\hline $\begin{array}{l}\text { F. semitectum Berkeley and } \\
\text { Ravenel }\end{array}$ & - & - & - & - & - & - & - & - & - & - & - & - \\
\hline F. solani (Mart.) Saccardo & + & + & . & . & . & . & . & . & . & . & . & + \\
\hline $\begin{array}{ll}\text { F. verticillioides } & \text { Sacc. } \\
\text { Nirenberg } & \end{array}$ & - & - & - & - & - & - & & & & + & & \\
\hline Mucor & . & + & . & + & + & + & + & + & . & - & - & . \\
\hline M. circinelloides Van Tieghem & - & + & - & & - & - & - & - & - & - & - & - \\
\hline M. hiemalis Wehmer & - & 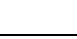 & - & + & + & - & + & & - & - & - & - \\
\hline M. racemosus Fresenius & - & + & - & + & - & + & - & + & - & - & - & - \\
\hline Neurospora & - & - & - & - & - & - & - & - & - & - & - & - \\
\hline N. crassa Shear and Dodge & - & - & - & - & - & - & - & - & - & - & - & - \\
\hline Penicillium & - & + & + & - & - & - & + & - & + & + & + & - \\
\hline P. chrysogenum Thom & - & - & & - & - & - & - & - & - & - & - & - \\
\hline P. corylophilum Dierckx & - & - & + & - & - & - & - & - & - & - & - & - \\
\hline P. cyclopium Westling & - & - & - & - & - & - & - & - & - & - & - & - \\
\hline P. digitatum (Pers. Ex Fr.) Sacc. & - & + & - & - & - & - & - & - & - & + & & - \\
\hline P. duclauxii Delacroix & - & + & - & - & - & - & - & - & - & - & + & - \\
\hline P. expansum Link ex Gray & - & - & - & - & - & - & - & - & - & - & - & - \\
\hline p. italicum Wehmer & - & - & - & - & - & - & - & - & - & - & - & - \\
\hline P. purpurogenum Stoll & - & + & - & - & - & - & - & - & - & - & - & - \\
\hline P. rugulosum Thom & - & - & - & - & - & - & - & - & - & - & - & - \\
\hline P. oxalicum Currie and Thom & - & - & - & - & - & - & + & - & + & - & - & - \\
\hline P. vermiculatum Dangeard & - & - & - & - & . & - & - & - & - & - & - & - \\
\hline Rhizopus & . & . & . & . & . & . & . & . & + & - & . & . \\
\hline R. oryzae Went \& Geerlings & - & - & & - & - & - & - & - & + & - & - & - \\
\hline Stachybotrys & + & - & + & - & - & - & - & . & + & . & - & - \\
\hline S. chartarum (Ehrenb.) Hughes & + & - & + & - & . & . & . & . & + & . & . & - \\
\hline Trichoderma & - & - & - & - & - & - & - & + & - & - & - & - \\
\hline T. hamatum (Bonord.) Bain & . & - & . & - & . & . & . & & - & . & - & . \\
\hline T. gansus yumenensis & - & - & - & - & - & - & - & + & - & - & - & - \\
\hline Trichurus & - & - & . & - & . & + & - & - & . & . & - & - \\
\hline T. spiralis Hasselbr & - & - & . & - & . & + & . & . & . & . & . & . \\
\hline Ulocladium & - & - & - & - & . & . & . & . & + & . & . & . \\
\hline U. atrum Preuss & . & - & - & - & . & - & . & - & + & - & . & - \\
\hline Total genera (13) & 2 & 5 & 4 & 2 & 3 & 3 & 3 & 5 & 5 & 3 & 3 & 2 \\
\hline Total species (57) & 2 & 15 & 7 & 6 & 4 & 3 & 3 & 6 & 7 & 3 & 5 & 4 \\
\hline Total varieties (2) & - & - & - & 2 & - & - & - & - & - & 1 & 1 & - \\
\hline
\end{tabular}

Table (1): continued. 


\begin{tabular}{|c|c|c|c|c|}
\hline \multirow[t]{2}{*}{ Fungal species and variety } & \multirow[t]{2}{*}{ Isolate No. } & \multirow[t]{2}{*}{ Source } & \multicolumn{2}{|c|}{ Mycotoxin production } \\
\hline & & & Kind of Toxin & "Level \\
\hline Acremonium rutilum & 1 & Spud & - & $\mathrm{N}$ \\
\hline Acremonium strictum & 2 & Canned apple juice & Zearalenone & $\mathrm{H}$ \\
\hline Alternaria alternata & 3 & Orange & Alternariol & $\mathrm{H}$ \\
\hline A. alternata & 4 & Orange juice & Alternariol & $\mathrm{H}$ \\
\hline A. alternata & 5 & Pomegranate & Alternariol & $\mathrm{H}$ \\
\hline A. alternata & 6 & Tomato juice & - & $\mathrm{N}$ \\
\hline A. brassicicol & 7 & Orange juice & Alternariol & $\mathrm{H}$ \\
\hline A. longipes & 8 & Orange & Alternariol & $\mathrm{H}$ \\
\hline A. solani & 9 & Tomato & Alternariol & $\mathrm{H}$ \\
\hline A. solani & 10 & Tomato juice & - & $\mathrm{N}$ \\
\hline Aspergillus awamorii & 11 & Canned guava juice & - & $\mathrm{N}$ \\
\hline A. awamorii & 12 & Lentils & - & $\mathrm{N}$ \\
\hline A. awamorii & 13 & Maize & - & $\mathrm{N}$ \\
\hline A. awamorii & 14 & Phaseolus & - & $\mathrm{N}$ \\
\hline A. awamorii & 15 & Spud & - & $\mathrm{N}$ \\
\hline A. caespitosus & 16 & Orange & Sterigmatocystin & $\mathrm{L}$ \\
\hline A. ficuum & 17 & Canned guava juice & - & $\mathrm{N}$ \\
\hline A. ficuum & 18 & Lunchoan & - & $\mathrm{N}$ \\
\hline A. ficuum & 19 & Orange & - & $\mathrm{N}$ \\
\hline A. ficuum & 20 & Peanuts & - & $\mathrm{N}$ \\
\hline A. ficuum & 21 & Pineapple & - & $\mathrm{N}$ \\
\hline A. ficuum & 22 & Strawberry & - & $\mathrm{N}$ \\
\hline A. ficuum & 23 & Tomato & - & $\mathrm{N}$ \\
\hline A. flavo-forcatis & 24 & Lunchaon & Aflatoxin $B_{1}$ & M \\
\hline A. flavo-forcatis & 25 & Maize & - & $\mathrm{N}$ \\
\hline
\end{tabular}

Table (2): Mycotoxins screening potential of 150 different isolates of filamentous fungi isolated from foodstuffs in Sohag Governorate, Egypt.

\begin{tabular}{|c|c|c|c|c|}
\hline \multirow{2}{*}{ Fungal species and variety } & \multirow{2}{*}{ Isolate No. } & \multirow[t]{2}{*}{ Source } & \multicolumn{2}{|c|}{ Mycotoxin production } \\
\hline & & & Kind of Toxin & "Level \\
\hline A. flavus & 26 & Bananas & - & $\mathrm{N}$ \\
\hline A. flavus & 27 & Broad bean & - & $\mathrm{N}$ \\
\hline A. flavus & 28 & Canned guava juice & Aflatoxins $B_{1}, G_{1}$ & $\mathrm{~L}$ \\
\hline A. flavus & 29 & Canned orange juice & Aflatoxin $B_{1}$ & $\mathrm{~L}$ \\
\hline A. flavus & 30 & Lemon & Aflatoxins $\mathrm{B}_{1}, \mathrm{~B}_{2}, \mathrm{G}_{1}, \mathrm{G}_{2}$ & $\mathrm{H}$ \\
\hline A. flavus & 31 & Lentils & Aflatoxins $\mathrm{B}_{1}, \mathrm{G}_{1}$ & $\mathrm{M}$ \\
\hline A. flavus & 32 & Lunchoan & Aflatoxins $\mathrm{B}_{1}, \mathrm{~B}_{2}, \mathrm{G}_{1}, \mathrm{G}_{2}$ & $\mathrm{M}$ \\
\hline A. flavus & 33 & Maize & - & $\mathrm{N}$ \\
\hline A. flavus & 34 & Orange & Aflatoxins $\mathrm{G}_{1}, \mathrm{G}_{2}$ & $\mathrm{~L}$ \\
\hline A. flavus & 35 & Peanuts & Aflatoxins $\mathrm{B}_{1}, \mathrm{G}_{1}$ & M \\
\hline A. flavus & 36 & Peanuts & - & $\mathrm{N}$ \\
\hline A. flavus & 37 & Phaseolus & Aflatoxins $B_{1}, B_{2}$ & $\mathrm{M}, \mathrm{L}$ \\
\hline A. flavus & 38 & Phaseolus & Aflatoxins $B_{1}, G_{1}$ & $\mathrm{M}$ \\
\hline A. flavus & 39 & Phaseolus & - & $\mathrm{N}$ \\
\hline A. flavus & 40 & Pomegranate & - & $\mathrm{N}$ \\
\hline A. flavus & 41 & Rice & - & $\mathrm{N}$ \\
\hline A. flavus & 42 & Tomato & Aflatoxins $\mathrm{B}_{1}, \mathrm{~B}_{2}, \mathrm{G}_{1}$, & $\mathrm{H}$ \\
\hline A. flavus & 43 & Tomato juice & Aflatoxins $\mathrm{B}_{1}, \mathrm{G}_{1}$ & $\mathrm{M}$ \\
\hline A. flavus & 44 & Wheat & Aflatoxins $B_{1}, G_{1}$ & $\mathrm{~L}$ \\
\hline A. flavus var. columnaris & 45 & Bananas & Aflatoxins $B_{1}, G_{1}$ & $\mathrm{~L}$ \\
\hline A. flavus var. columnaris & 46 & Canned guava juice & - & $\mathrm{N}$ \\
\hline A. flavus var. columnaris & 47 & Canned guava juice & Aflatoxin $B_{1}$ & $\mathrm{~L}$ \\
\hline A. flavus var. columnaris & 48 & Canned orange juice & Aflatoxin $\mathrm{B}_{1}$ & $\mathrm{~L}$ \\
\hline A. flavus var. columnaris & 49 & Canned orange juice & Aflatoxins $\mathrm{B}_{1}, \mathrm{G}_{1}$ & M \\
\hline A. flavus var. columnaris & 50 & Lemon & Aflatoxin $\mathrm{B}_{1}$ & $\mathrm{~L}$ \\
\hline
\end{tabular}

Table (2): Continued.

\begin{tabular}{|c|c|c|c|c|}
\hline \multirow[t]{2}{*}{ Fungal species and variety } & \multirow[t]{2}{*}{ Isolate No. } & \multirow[t]{2}{*}{ Source } & \multicolumn{2}{|c|}{ Mycotoxin production } \\
\hline & & & Kind of Toxin & "Level \\
\hline A. flavus var. columnaris & 51 & Lentils & Aflatoxin $B_{1}$ & $\mathrm{M}$ \\
\hline A. flavus var. columnaris & 52 & Lunchaon & Aflatoxin $B_{1}$ & M \\
\hline A. flavus var. columnaris & 53 & Maize & Aflatoxin $B_{1}$ & M \\
\hline A. flavus var. columnaris & 54 & Orange juice & Aflatoxin $B_{1}$ & M \\
\hline A. flavus var. & 55 & Phaseolus & Aflatoxins $\mathrm{B}_{1}, \mathrm{~B}_{2}$ & $\mathrm{~L}$ \\
\hline A. flavus var. columnaris & 56 & Tangerine & Aflatoxins $\mathrm{B}_{1}$ & $\mathrm{~L}$ \\
\hline A. flavus var. columnaris & 57 & Tomato & - & $\mathrm{N}$ \\
\hline A. flavus var. columnaris & 58 & Tomato juice & - & $\mathrm{N}$ \\
\hline A. foetidus & 59 & Carrot & - & $\mathrm{N}$ \\
\hline A. foetidus & 60 & Lemon & - & $\mathrm{N}$ \\
\hline A. foetidus var. pallidus & 61 & Canned guava juice & - & $\mathrm{N}$ \\
\hline A. foetidus var. pallidus & 62 & Phaseolus & - & $\mathrm{N}$ \\
\hline A. fumigatus & 63 & Lemon & - & $\mathrm{N}$ \\
\hline A. fumigatus & 64 & Potato & - & $\mathrm{N}$ \\
\hline A. granulosus & 65 & Lemon & - & $\mathrm{N}$ \\
\hline A. heteromorphus & 66 & Bananas & - & $\mathrm{N}$ \\
\hline A. japonicus & 67 & Strawberry & - & $\mathrm{N}$ \\
\hline A. japonicus & 68 & Tomato & - & $\mathrm{N}$ \\
\hline A. nidulans & 69 & Orange & Sterigmatocystin & $\mathrm{H}$ \\
\hline A. niger & 70 & Lunchaon & - & $\mathrm{N}$ \\
\hline A. niger & 71 & Peanuts & - & $\mathrm{N}$ \\
\hline A. niger & 72 & Phaseolus & - & $\mathrm{N}$ \\
\hline A. niger & 73 & Strawberry & - & $\mathrm{N}$ \\
\hline A. niger & 74 & Tomato juice & - & $\mathrm{N}$ \\
\hline A. niger & 75 & Wheat & - & $\mathrm{N}$ \\
\hline
\end{tabular}

Table (2): Continued. 


\begin{tabular}{|c|c|c|c|c|}
\hline \multirow[t]{2}{*}{ Fungal species and variety } & \multirow[t]{2}{*}{ Isolate No. } & \multirow[t]{2}{*}{ Source } & \multicolumn{2}{|c|}{ Mycotoxin production } \\
\hline & & & Kind of Toxin & "Level \\
\hline A. ochraceus & 76 & Lemon & Ochratoxins A, B & $\mathrm{H}, \mathrm{M}$ \\
\hline A. parasiticus & 77 & Lunchaon & Aflatoxin $B_{1}$ & $\mathrm{M}$ \\
\hline A. parasiticus & 78 & Orange & Aflatoxin $B_{1}$ & $\mathrm{~L}$ \\
\hline A. parasiticus & 79 & Phaseolus & Aflatoxins $\mathrm{B}_{1}, \mathrm{~B}_{2}$ & $\mathrm{~L}$ \\
\hline A. phonicis & 80 & Canned apple juice & - & $\mathrm{N}$ \\
\hline A. phonicis & 81 & Tangerine & - & $\mathrm{N}$ \\
\hline A. pulverulentus & 82 & Lemon & - & $\mathrm{N}$ \\
\hline A. pulvinus & 83 & Peanuts & - & $\mathrm{N}$ \\
\hline A. sclerotiorum & 84 & Spud & Ochratoxins A, B & $\mathrm{H}$ \\
\hline A. sulphureus & 85 & Lemon & Ochratoxins A, B & M \\
\hline A. sydowii & 86 & Bananas & - & $\mathrm{N}$ \\
\hline A. sydowii & 87 & Canned orange juice & - & $\mathrm{N}$ \\
\hline A. sydowii & 88 & Lunchaon & - & $\mathrm{N}$ \\
\hline A. sydowii & 89 & Tomato juice & - & $\mathrm{N}$ \\
\hline A. terreus & 90 & Canned apple juice & - & $\mathrm{N}$ \\
\hline A. terreus & 91 & Canned orange juice & - & $\mathrm{N}$ \\
\hline A. terreus & 92 & Orange & - & $\mathrm{N}$ \\
\hline A. terreus & 93 & Tomato juice & - & $\mathrm{N}$ \\
\hline A. tubingensis & 94 & Bananas & - & $\mathrm{N}$ \\
\hline A. tubingensis & 95 & Canned apple juice & - & $\mathrm{N}$ \\
\hline A. tubingensis & 96 & Canned orange juice & - & $\mathrm{N}$ \\
\hline A. tubingensis & 97 & Lemon & - & $\mathrm{N}$ \\
\hline A. tubingensis & 98 & Lunchaon & - & $\mathrm{N}$ \\
\hline A. tubingensis & 99 & Orange & - & $\mathrm{N}$ \\
\hline A. tubingensis & 100 & Pomegranate & - & $\mathrm{N}$ \\
\hline A. versicolor & 101 & Canned guava juice & - & $\mathrm{N}$ \\
\hline Drechslera rostrata & 102 & Lemon & Alternariol & $\mathrm{M}$ \\
\hline
\end{tabular}

Table (2): Continued.

\begin{tabular}{|c|c|c|c|c|}
\hline \multirow[t]{2}{*}{ Fungal species and variety } & \multirow[t]{2}{*}{ Isolate No. } & \multirow[t]{2}{*}{ Source } & \multicolumn{2}{|c|}{ Mycotoxin production } \\
\hline & & & Kind of Toxin & "Level \\
\hline $\begin{array}{c}\text { Fusarium equiseti } \\
\text { F. equiseti } \\
\text { F. proliferatum } \\
\text { F. reticulatum } \\
\text { F. semitectum } \\
\text { F. solani } \\
\text { F. solani } \\
\text { F. solani } \\
\text { F. solani } \\
\text { F. solani } \\
\text { F. verticillioides } \\
\text { F. verticillioides } \\
\text { Mucor circinelloides } \\
\text { M. hiemalis } \\
\text { M. hiemalis } \\
\text { M. hiemalis } \\
\text { M. racemosus } \\
\text { M. racemosus } \\
\text { M. racemosus } \\
\text { M. racemosus } \\
\text { M. racemosus } \\
\text { M. racemosus } \\
\text { Nurospora crassa } \\
\text { Penicillium chrysogenum } \\
\text { P. corylophilum } \\
\text { P. corylophilum } \\
\text { P. cyclopium }\end{array}$ & \begin{tabular}{|l|}
103 \\
104 \\
105 \\
106 \\
107 \\
108 \\
109 \\
110 \\
111 \\
112 \\
113 \\
114 \\
115 \\
116 \\
117 \\
118 \\
119 \\
120 \\
121 \\
122 \\
123 \\
124 \\
125 \\
126
\end{tabular} & $\begin{array}{l}\text { Carrot } \\
\text { Wheat } \\
\text { Spud } \\
\text { Peanuts } \\
\text { Orange juice } \\
\text { Maize } \\
\text { Millet } \\
\text { Orange } \\
\text { Orange juice } \\
\text { Wheat } \\
\text { Maize } \\
\text { Tangerine } \\
\text { Orange } \\
\text { Phaseolus } \\
\text { Pomegranate } \\
\text { Rice } \\
\text { Bananas } \\
\text { Lunchoan } \\
\text { Orange } \\
\text { Phaseolus } \\
\text { Potato } \\
\text { Spud } \\
\text { Broad bean } \\
\text { Lemon } \\
\text { Bananas } \\
\text { Peanuts } \\
\text { Bananas }\end{array}$ & $\begin{array}{c}- \\
- \\
- \\
\text { Diacetoxyscirpenol } \\
\text { Zearalenone } \\
- \\
- \\
- \\
- \\
\text { Fuminisins } \mathrm{B}_{1}, \mathrm{~B}_{2} \\
- \\
- \\
- \\
- \\
- \\
- \\
- \\
- \\
- \\
- \\
- \\
- \\
- \\
- \\
\text { Patulin } \\
\text { Patulin } \\
\text { Patulin }\end{array}$ & 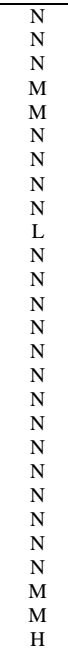 \\
\hline
\end{tabular}

Table (2): Continued.

\begin{tabular}{|c|c|c|c|c|}
\hline \multirow[t]{2}{*}{ Fungal species and variety } & \multirow[t]{2}{*}{ Isolate No. } & \multirow[t]{2}{*}{ Source } & \multicolumn{2}{|c|}{ Mycotoxin production } \\
\hline & & & Kind of Toxin & "Level \\
\hline P. digitatum & 130 & Orange & - & $\mathrm{N}$ \\
\hline P. digitatum & 131 & Tangerine & Patulin & $\mathrm{H}$ \\
\hline P. duclauxii & 132 & Orange & Patulin & $\mathrm{H}$ \\
\hline P. duclauxii & 133 & Pineapple juice & - & $\mathrm{N}$ \\
\hline P. duclauxii & 134 & Tomato & - & $\mathrm{N}$ \\
\hline P. expansum & 135 & Bananas & Patulin & $\mathrm{H}$ \\
\hline P. expansum & 136 & Lemon & - & $\mathrm{N}$ \\
\hline p. italicum & 137 & Lemon & Patulin & $\mathrm{M}$ \\
\hline P. purpurogenum & 138 & Orange & - & $\mathrm{N}$ \\
\hline P. rugulosum & 139 & Carrot & - & $\mathrm{N}$ \\
\hline P. oxalicum & 140 & Rice & - & $\mathrm{N}$ \\
\hline P. oxalicum & 141 & Strawberry & - & $\mathrm{N}$ \\
\hline P. vermiculatum & 142 & Lunchoan & Patulin & $\mathrm{H}$ \\
\hline Rhizopus oryzae & 143 & Strawberry & - & $\mathrm{N}$ \\
\hline Stachybotrys charatarum & 144 & Millet & Zearalenone & M \\
\hline S. charatarum & 145 & Peanuts & Zearalenone & $\mathrm{M}$ \\
\hline S. charatarum & 146 & Strawberry & - & $\mathrm{N}$ \\
\hline Trichoderma hamatum & 147 & Canned orange juice & - & $\mathrm{N}$ \\
\hline T. gansus & 148 & Spud & - & $\mathrm{N}$ \\
\hline Trichurus spiralis & 149 & Potato & - & $\mathrm{N}$ \\
\hline Ulocladium atrum & 150 & Strawberry & - & $\mathrm{N}$ \\
\hline
\end{tabular}

Table (2): Continued. 
* The level of mycotoxin production:

$\mathrm{H}=$ High level; more than $500 \mu \mathrm{g} / \mathrm{L}$ medium.

$\mathrm{M}=$ Moderate level; ranged from 200- 500 $\mu \mathrm{g} / \mathrm{L}$ medium.

$\mathrm{L}=$ Low level; less than $200 \mu \mathrm{g} / \mathrm{L}$ medium. $\mathrm{N}=$ No detectable toxin level.

\section{References:}

Abdel-Kareem, M.M. (2010). Microbiota and mycotoxins occurrence in some Egyptian fruit juices. M Sc Thesis, Department of Botany, Faculty of Science, Sohag University, Sohag, Egypt.

Ainsworth, G.C. (1971). Ainsworth and Bishy's Dictionary of fungi: (P. 412) Commonwealth Mycological Institute, Kew, Surry, England.

Alborch, L., Bragulat, M.R., Castellá, G., Abarca, M.L. and Cabañes, F.J. (2012). Mycobiota and mycotoxin contamination of maize flours and popcorn kernels for human consumption commercialized in Spain. Food Microbiology. 32:97-103.

Al-Doory, Y. (1980). Laboratory Medical Mycology. London Lea and Febiger, p. 410.

Ariňo, A., Herrera, M., Estopaňan, G., Rota, M.C., Carramiňana, J.J. and Juan, T. (2009). Aflatoxins in bulk and pre-packed pistachios sold in Spain and effect of roasting. Food Control. 20: 811-814.

Bennett, J.W. and Klich, M. (2003). Mycotoxins. Clin. Microbiol. Rev. 16: 497-516.

Bennett, J.W. Henderberg, A. and Grossman, K. (1989). Sterigmatocystin production on complex and defined substrates. Mycopathologia 105: 35 - 38.

Booth, C. (1977). Fusarium laboratory guide to the indentification of the major species. (P. 58) Commonwealth Mycological Institute, Kew, Surrey, England.

CAST, (2003). Mycotoxins: Risks in Plant, Animal and Human Systems. Report No. 139. Council for Agricultural Science and Technology, Ames, Iowa, USA.
Chelkowski, J. (editor), (1991). Cereal grain. Mycotoxins, Fungi and Quality in Drying and Storage.Elsevier, Amsterdam.

Dantigny, P., Guilmart, A. and Bensoussan, M. (2005). Basis of predictive mycology. Int. J. Food Microbiol. 100: 187-196.

Delage, N., Harlingue, A.d., Colonna Ceccaldi, B. and Bompeix, G. (2003). Occurrence of mycotoxins in fruit juices and wine. Food Control 14: 225 227.

Diener, U.L. and Davis, N.D. (1966). Aflatoxin production by isolates of Aspergillus flavus. Phytopathology 56: 1390 129.

Domsch, K.H., Gams, W. and Anderson, T. (2007). Compendium of fungi. $2^{\text {nd }}$ Edition, IHW Verlag, Eching, Germany.

Dong, Z., Liu, G., Dong, Z., Qian, Y., An, Y., Miao, J. and Zhen, Y. (1987). Carcinogenesis 8: 989.

El-Maraghy, S.S. and Zohri, A.A. (1988). Mycotoxin - producing potential of aspergilli and penicillia of broad beans in Egypt. Bull. Faculty of Science, Assiut Univ. Egypt 17: 91 - 102.

El-Maghraby, O.M., El-Kady, I.A. and Soliman, S. (1995). Mycoflora and Fusarium toxins of three types of corn grains in Egypt with special reference to production of trichothecene-toxins. Microbiol. Res. 150 (3): 225-232.

El-Kady, I.A. and Moubasher, M.H. (1982). Toxigenicity and toxin of Stachybotrys chartarum isolates from wheat straw samples in Egypt. Experimental Mycology 6: $25-31$.

Ellis, M.B. (1976). More Dematiaceous Hyphomycetes. (p. 507) Commonwealth Mycological Institute, Kew, Surrey, England.

Filtenborg, O., Frisvad, J.C. and Thrane, U. (1996). Moulds in food spoilage. International Journal of Food Microbiology. 33: 85-102.

Gimeno, A. (1979). Thin-layer chromatographic determination of 
aflatoxins, ochratoxins, sterigmatocystin, zearalenone, citrinin, T-2 toxin, diacetoxyscirpenol, penicillic acid and penitrem "A". J. Assoc. Off. Anal. Chem. 62: (579- 585).

Huis in't Veld, J.H.J. (1996). Microbial and biochemical spoilage of foods: an overview.Int.J.Food Microbiol. 33: 1-18.

Klich, M.A. and Pitt, J.I. (1992). A laboratory guide to the common Aspergillus species and their teleomorphs. Common Wealth Scientific and Industrial Research Organization, Division of Food Processing, North Ryde, Australia.

Köppen, R., Koch, M., Siegel, D., Merkel, S. and Maul, R. (2010). Determination of mycotoxins in foods: current state of analylical methods and limitations. Applied Microbiology and Biotechnology, New York. 86:1595-1612.

Lillehoj, E.B. and Ciegler, A. (1968). Biological activity of sterigmatocystin. Mycopathol. Mycol. Appl. 35: 373 - 376.

Marasas, W.F.O., Nelson, P.E. and Tousson, T.A. (1984). Toxtgenic Fusanum species. identity and mycotoxicology. University Park, PA: Pennsylvania State University Press 350.

Medeiros, F.H.V., Martins, S.J. and Zucchi, T.D. (2012). Biological control of mycotoxin-producing molds. Ciênc. agrotec.36 (5): 483-497.

Moubasher, A.H. (1993). Soil fungi in Qatar and other Arab countries. Published by the Scientific and Applied Rrsearch Center, University of Qatar, Qatar.

Miller, D.M. and Wilson, D.M. (1994). Veterinary diseases related to aflatoxins. In Eaton, D. L. and Groopman, J. D. (Eds.). The Toxicology of Aflatoxins. Academic Press, Inc., New York, pp. 347-364.

Nelson, P.E., Desjardins, A.E. and Plattner, R.D. (1993). Ann. Rev. Phytopathol. 31: 233 - 252. Cited in Aboul-Enien, A. M.; Amra, H.
A., Ragab, A. A. and Mohamed, M. I. (2000): The occurrence of fumonisin $\mathrm{B}_{1}$ in Egyptian market corn, screening, processing and toxicology. First Symposium on Food Safety, Oct. 9 - 11, Coll. Agri. \& Food Sci., King Faisal Univ. Saudi Arabia, Proceedings $256-271$.

Njobeh, P.B., Dutton, M.F., Koch, S.H., Chuturgoon, A., Stoev, S. and Seifert, K. (2009): Contamination with storage fungi of human food from Cameroon. International Journal of Food Microbiology. 135: 193-198.

Obied-Allah, M.R. (2011). Mycotoxigenic Fusarium species and natural occurrence of fumonisin in corn (Zea mays L.) in Sohag Governorate. MSc Thesis, Department of Botany, Faculty of Science, Sohag University, Sohag, Egypt.

Pitt, J.I. (1979). The genus Penicullium and its teleomorphic states Eupenicillium and Talaromyces. (p. 634) Academic Press. Inc. (London).

Pitt, J.I. (1996): What are mycotoxins? Aus. Mycotoxin Newslett.7: 1.

Pitt, J.I., Hocking, A.D., Samson, R.A. and King, A.D. (1992). Recommended methods for mycological examination of foods, 1992. In: Samson R. A., Hocking A. D., Pitt J. I., and King A. D. (editors), Modern Methods in Food Mycology. Elsevier, Amsterdam, pp. 365-368.

Pitt, J.I. and Hocking, A.D. (2009). Fungi and food spoilage. $3^{\text {rd }}$ Edition, Spinger Science and Business Media.

Raper, K.B. and Fennel, P.I. (1965). The genus Aspergillus (p. 686) Williams and Wilkins Baltimore U. S. A.

Reddy, K.R.N., Reddy, C.S. and Muralidharan, K. (2009). Detection of Aspergillus spp. and aflatoxin $B_{1}$ in rice in India. Food Microbiology, 26, 27-31.

Richard, J.L. (2007). Some major mycotoxins and their 
mycotoxicosis - An overview. International Journal of Food Microbiology, Amsterdam.119: 3-10.

Ruadrew, S., Craft, J. and Aidoo, K. (2013). Occurrence of toxigenic Aspergillus spp. and aflatoxins in selected food commodities of Asian origin sourced in the West of Scotland. Food and Chemical Toxicology. 55: 653-658.

Sánchez-Hervás, M., Gil, J.V., Bisbal, F., Ramón, D. and MartínezCulebras, P.V. (2008). Mycobiota and mycotoxin producing fungi from cocoa beans. International Journal of Food Microbiology 125: $336-340$.

Schrader, T.J., Cherry, W., Soper, K., Langlois, I. and Vijay, H.M. (2001). Teratogen. Carcinogen. Mutagen.21: 261.

Schroeder, H.W. and Kelton, W.H. (1975). Production of sterigm- atocystin by some species of the genus Aspergillus flavus and its toxicity to chicken embryos. Appl. Microbiol 50: 583 - 585 .

Scott, P.M., Lawrence, J.W. and Van Walbeak, W. (1970). Detection of mycotoxins by thin-layer chromatography: Application to screening of fungal extracts. Appl. Microbiol 20: 839 - 842.

Tipples, K.H. (1995). Quality and nutrItional changes in stored grain. In: Jnyas, D. S.; White, N. D. G. and Muir, W. E. (editors). Stored Grain Ecosystems. Marcel Dekker. Neh York. pp. 325-351.

Toffa, D.D., Mahnine, N., Ouaffak, L., El Abidi, A., El Alaoui Faris, F.Z. and Zinedine, A. (2013). First survey on the presence of ochratoxin A and fungi in raw cereals and peanut available in the Republic of Niger. Food Control. 32: 558-562.

Tournas, V.H., Heeres, J. and Burgess, L. (2006). Moulds and yeasts in fruit salads and fruit juices. Food Microbiology 23: $684-688$.

Youssef, M.S. (1986). Mycoflora and mycotoxin of soybean seeds in Egypt. MSc Thesis, Department of Botany, Faculty of Science, Sohag University, Sohag, Egypt.

Varga, J., Frisvad, J.C. and Samson, R.A. (2011). Two new aflatoxins producing species, and an overview of Aspergillus section Flavi. Studies in Mycology. 69: 57-80.

Zohri, A.A. and Ismail, M.A. (1994). Based on biochemical and physiological behavior, where is Aspergillus egyptiacus better placed? .Folia Microbiol. 39 (5): 415 - 419.

Zohri, A.A., Saber, S.M. and Abdel-Gawad, K.M. (1992). Fungal flora and mycotoxins associated with onion (Allium cepa L.) in Egypt. Kor. J. Mycol. 20: 302-308.

Zohri, A.A., Abdel-Sater, M.A. and Ismail, M.A. (1995). Incidence of aflatoxins and mould flora in corn snacks. J. Food Science \& Technology. 32 (4): 289-294.

Zucchi, T.D. and Melo, I.S. (2009). Controle biológico de fungos aflatoxigênicos. In: Bettiol, W. and Morandi, M. A. B. editores. Biocontrole de Doenças de Plantas: Uso e Perspectivas. 1: 69-74.

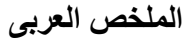

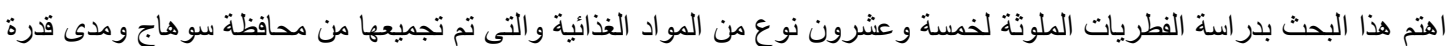

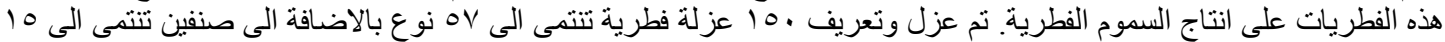

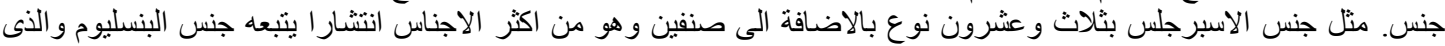

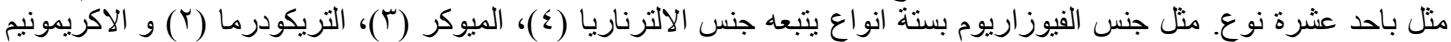

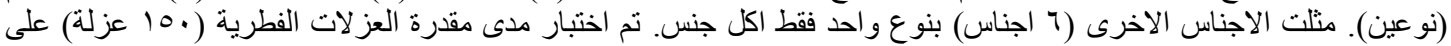

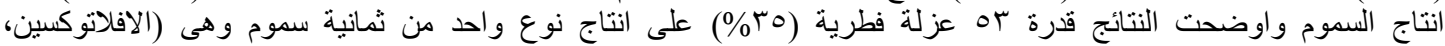

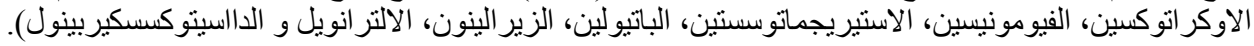

